Received: 23 June 2017

Accepted: 4 August 2017

Published online: 29 August 2017

SCIENTIFIC REP

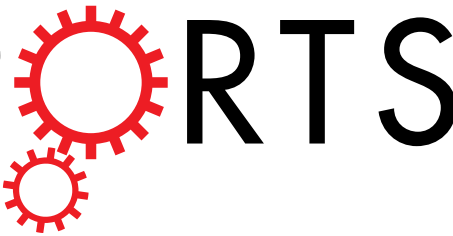

OPEN

\title{
Chitin-deacetylase activity induces appressorium differentiation in the rice blast fungus Magnaporthe
}

oryzae

Misa Kuroki ${ }^{1}$, Kana Okauchi ${ }^{1}$, Sho Yoshida ${ }^{1}$, Yuko Ohno ${ }^{1}$, Sayaka Murata ${ }^{1}$, Yuichi Nakajima ${ }^{2}$, Akihito Nozaka ${ }^{1}$, Nobukiyo Tanaka ${ }^{1}$, Masahiro Nakajima ${ }^{1}$, Hayao Taguchi ${ }^{1}$, Ken-ichiro Saitoh $\mathbb{D}^{3}$, TohruTeraoka ${ }^{4}$, Megumi Narukawa ${ }^{1}$ \& Takashi Kamakura ${ }^{1}$

The rice blast fungus Magnaporthe oryzae differentiates a specialized infection structure called an appressorium to invade rice cells. In this report, we show that $C B P 1$, which encodes a chitindeacetylase, is involved in the induction phase of appressorium differentiation. We demonstrate that the enzymatic activity of $\mathrm{Cbp} 1$ is critical for appressorium formation. $M$. oryzae has six CDA homologues in addition to Cbp1, but none of these are indispensable for appressorium formation. We observed chitosan localization at the fungal cell wall using $\mathrm{OGA}^{488}$. This observation suggests that $\mathrm{Cbp1-}$ catalysed conversion of chitin into chitosan occurs at the cell wall of germ tubes during appressorium differentiation by $M$. oryzae. Taken together, our results provide evidence that the chitin deacetylase activity of Cbp1 is necessary for appressorium formation.

The worldwide rice harvest is approximately 500 million tonnes per year ${ }^{1}$, but $10-15 \%$ of this total yield is lost due to disease $e^{2}$. One of the major causes of disease losses in rice is the fungus Magnaporthe oryzae, which causes rice blast. Rice leaves infected by $M$. oryzae display necrotic lesions and heavy infections can kill rice seedlings. The infection process of $M$. oryzae is initiated by the attachment of a conidium to the rice surface through the release of spore tip mucilage ${ }^{3}$. After germination in a water droplet on the rice leaf, the conidium elaborates a germ tube ${ }^{4}$, which begins to form a dome-shaped appressorium at its $\operatorname{tip}^{5-7}$. The fungus then generates enormous turgor pressure in the appressorium and produces a penetration peg that pierces the plant cell wall to enter an epidermal cell $^{8,9}$. The appressorium is a specialized infection structure that is crucial for host plant penetration ${ }^{10}$. Therefore, knowledge of the mechanism of appressorium formation may contribute to disease control.

Appressorium development can be induced by several physical and chemical factors; surface hydrophobicity ${ }^{11,12}$, the hardness of the contact surface ${ }^{13,14}$ and cutin monomers released from the plant surface ${ }^{15}$. Signal transduction pathways such as cAMP signalling and mitogen-activated protein kinase cascades are required for appressorium morphogenesis ${ }^{6,10}$.

Kamakura et al. ${ }^{16}$ previously reported that the gene $C B P 1$, which encodes a Chitin-Binding Protein (MGG_12939.7), is involved in appressorium formation by $M$. oryzae. CBP1 is specifically expressed in germlings and the null mutant of $C B P 1$ shows delayed appressorium differentiation on hydrophobic surfaces. Eight hours post inoculation (hpi), the frequency of appressorium formation in the $\Delta c b p 1$ mutant was very low, but increased to the same level as in an isogenic wild type strain at $24 \mathrm{hpi}$ on hydrophobic artificial substrates. The $\Delta c b p 1$ mutant also retains the ability to cause disease symptoms on leaves of susceptible rice cultivars ${ }^{16}$. When conidia of $\Delta c b p 1$ were treated with 3-isobutyl-1-methylxanthine, which induces intracellular accumulation of $\mathrm{cAMP}^{17}$, or 1,16-hexadecanediol (HDD), a minor component of cutin ${ }^{15}$, they were able to form appressoria on artificial

\footnotetext{
${ }^{1}$ Tokyo University of Science, Department of Applied Biological Science, Faculty of Science and Technology, 2641, Yamazaki, Noda, Chiba, 278-8510, Japan. ${ }^{2}$ Nagoya University, Graduate School of Bioagricultural Sciences, School of Agricultural Sciences, Furo-cho, Chikusa, Nagoya, Aichi, 464-8601, Japan. ${ }^{3}$ Tokyo University of Agriculture and Technology, University Research Administration Center, 2-24-16, Naka-cho, Koganei, Tokyo, 184-8588, Japan. ${ }^{4}$ Tokyo University of Agriculture and Technology, Institute of Symbiotic Science and Technology, 3-5-8, Saiwai-cho, Fuchu, Tokyo, 183-8509, Japan. Correspondence and requests for materials should be addressed to T.K. (email: kamakura@rs.noda.tus.ac.jp)
} 
(a)

Magnaporthe oryzae Metarhizium anisopliae Colletotrichum lindemuthianum Aspergillus nidulans Flammul ina velutipes Mucor rouxit Saccharomyces cerevisiae
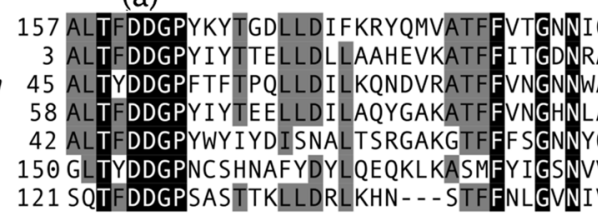
GKGMINDRTKPWAGYIQRMIAE RAKGHIDDPATEWPS I LRRMYNA WAN - - - I IEAGSNPDT I RRMRAD LAG - . - . - NEWL IQRVVNEG YQR - - - I I DQGAIDQIKYAYNA VDD - - - - WPYGAMRGVVDG

(b)

Magnaporthe oryzae Metarhizium anisopliae

Colletotrichum lindemuthianum Aspergillus nidulans Flammulina velutipes Mucor rouxii

Saccharomyces cerevisiae

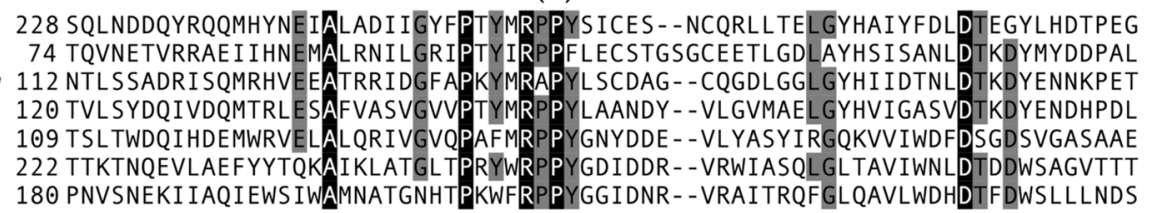

Magnaporthe oryzae Metarhizium anisopliae 297 AQIGKDIWDKAVGP - - ANPADRSFLQIEHDLQYQSVYNLTEYFLKSIQAKGFRSVPVGVCLGDDPANW̄YR 145 IQRSKDRYSSTLS - - - TNSKENSY IVLAHDVHEQTVHNLTEYMISLARERGYKLVTVGECLGDPEENWYR 181 THLSAEKFNNELS----ADVGANSYIVLSHI Aspergillus nidulans Flammul ina velutipes Mucor rouxi i Saccharomyces cerevisiae

189 IGRSVAKFNQELD--.--- QGGTIVLSH 178 SKERYRTTSLNRP-.-.-.-. - STLLALNHETIESTAHDVIPYAIDRLQSAGYRLVTVAECLGEQPYQWTG 291 VEAVEQSYSDY IAMGTNGTFANSGNIVLTHE INTTMSLAVENLPKI I SAYKQVID - - VATCYNISHPYFED 249 VITEQE ILQNVIN - - - - WNKSGSGL I LEHDSTEKTVDLAIKINKL I - - - -GDDQSTVSHCVGGIDYIKEF

Figure 1. Multiple alignment of protein sequences. The amino acid sequence of the CDA-homologous domain in Cbp1 was aligned with CDAs in other species. The CDA sequences included (GenBank accession number) KFG84684.1 from Metarhizium anisopliae, AAT68493.1 from Colletotrichum lindemuthianum, ACF22100.1 from Aspergillus nidulans, BAE92728.1 from Flammulina velutipes, CAA79525.1 from Mucor rouxii, and KZV09551.1 from Saccharomyces cerevisiae. White letters on a black background indicate residues perfectly conserved across all the sequences, black letters on a grey background indicate regions conserved in at least five species out of seven. (a) Is a group of amino acids (TFDD) that comprise the CDA active site, including two aspartate residues. (b) Is also part of the CDA active site; RPPY, including tyrosine.

hydrophobic surfaces at $8 \mathrm{hpi}$, as similarly to the wild type strain ${ }^{16}$. These results suggest that Cbp1 is not essential for appressorium formation and primary infection of rice leaves, but clearly indicate that Cbp 1 facilitates appressorium differentiation on artificial substrates. It was predicted that $\mathrm{Cbp} 1$ has a signal peptide sequence and Ser/Thr cluster and that the protein localizes at the fungal cell surface ${ }^{16}$. Recently, Geoghegan and Gurr ${ }^{18}$ observed cell-surface localization of $\mathrm{Cbp} 1$ through expression of a Cbp1-mCherry fusion protein. Cbp1 localizes to the periplasm in a similar way to the sensor of hydrophobicity Mpg $1^{19}$ and the MagB heterotrimeric G-protein catalytic subunit ${ }^{15}$. Furthermore, the amino acid sequence of Cbpl suggests it is a glycosyl phosphatidyl inositol (GPI)-anchored protein. Some GPI-anchored proteins are involved in signalling pathways, such as Ecm 33 in Aspergillus fumigatus ${ }^{20}$, and Pga31 and Pga62 in Candida albicans ${ }^{21}$. These characteristics of Cbp1 support a potential role for the protein as an upstream effector of a signal transduction pathway.

In the present work, we show that Cbp1 actually acts as a chitin-deacetylase (CDA) (consistent with the findings of $\left.{ }^{18}\right)$. CDA generally converts chitin into chitosan. Chitin is a linear $\beta$ - $(1,4)$-linked insoluble homopolymer of the acetylated amino sugar $\mathrm{N}$-acetylglucosamine (GlcNAc). Chitin is a major component of the cells of most filamentous fungi ${ }^{22}$ and contributes to the strength and integrity of the fungal cell wall and septum ${ }^{23}$. Chitosan, generated by deacetylation of chitin, is produced enzymatically by CDA, which hydrolyses the $N$-acetamido groups of GlcNAc residues in chitin ${ }^{24}$. CDA is not currently known to act as part of a signalling pathway in other species, so we investigated whether Cbp1 acts by a novel mechanism. Before now, CDA activity has only been observed indirectly and enzymatic CDA activity has not been observed in M. oryzae.

\section{Results}

$\mathrm{CDA}$ activity of $\mathrm{Cbp} 1$ is required for appressorium formation. CDA genes have previously been cloned and characterized from several fungi and insects. Multiple sequence alignments have demonstrated that they have five well-conserved catalytic motifs ${ }^{25}$. These motifs make up the CDA active site ${ }^{26}$ and are conserved in CDA genes such as those in Metarhizium anisopliae [KFG84684.1] ${ }^{27}$, Colletotrichum lindemuthianum $[\text { AAT68493.1] }]^{28}$, Aspergillus nidulans $\left[\right.$ ACF22100.1] ${ }^{29}$, Flammulina velutipes [BAE92728.1] ${ }^{30}$, Mucor rouxii [CAA79525.1 $]^{24}$ and Saccharomyces cerevisiae [KZV09551.1] ${ }^{31}$ (Figs 1 and S1). These proteins have been demonstrated to show CDA activity experimentally. We observed that Cbp1 possessed these motifs. To test whether Cbp1 had CDA activity, we generated CDA active site substitution mutants and observed their phenotypes. One of the conserved motifs (TFDD, Fig. 1(a)) includes two aspartic acid residues; one was reported to interact with zinc or cobalt and the second binds acetate released from the substrate ${ }^{26}$.

We chose the first aspartate residue in the (a) domain and replaced it with alanine. We used the pCold I vector and heterologously expressed Cbp1 and the Cbp1-D161A substitution mutant in Escherichia coli. The crude extract of the Cbp1-expression strain showed significantly higher CDA activity than those of the vector control and Cbp1-D161A-expression mutant (Fig. 2(a)). These data indicated that Cbp1 has activity as a CDA.

We then transformed $\Delta c b p 1$ M. oryzae using Cbp1-D161A and obtained a CDA-inactive Cbp1 mutant. We concurrently generated a wild type $C B P 1$-complemented strain. The ability to elaborate appressoria was tested 

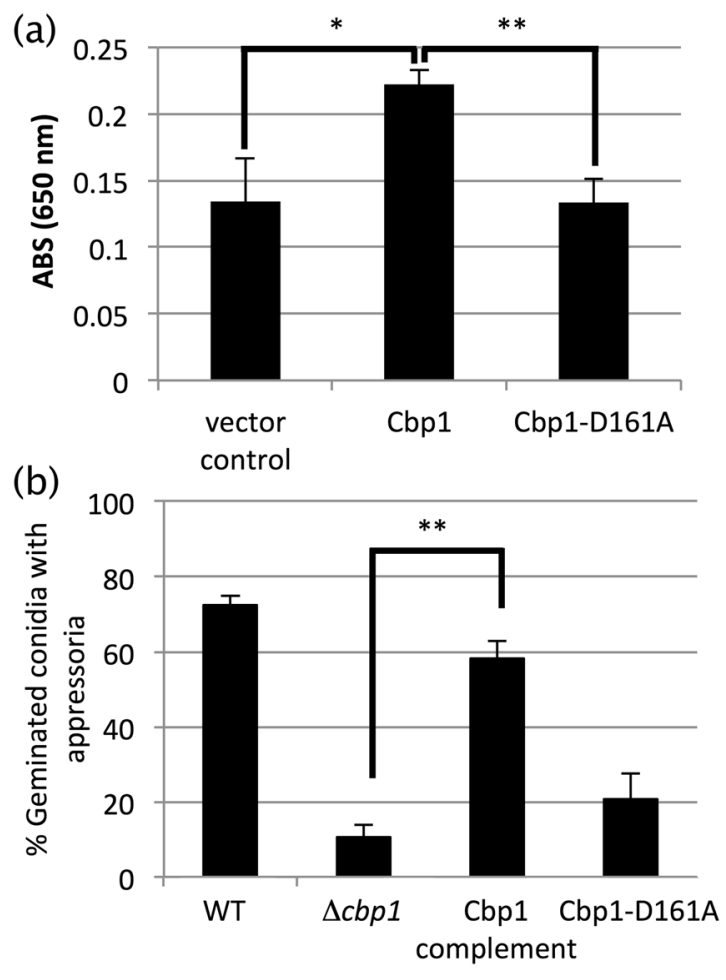

Figure 2. Effect of point mutation of Cbp1 on CDA activity and appressorium formation. Cbp1 and Cbp1D161A were compared for CDA activity and appressorium formation. Cbp1-D161A has a point mutation changing the first aspartate residue in the (a) region in Fig. 1 to alanine. (a) The CDA activity of crude extract expressed in E. coli was observed by the MBTH method. The experiment was performed in triplicate for each sample and repeated four times. ${ }^{*} p<0.05, * * p<0.01$ (Student's $t$-test) compared with Cbp1. Error bars indicate standard error. (b) Appressorium formation rates were scored at $6 \mathrm{~h}$ post inoculation (hpi) on hydrophobic polyvinyl chloride (PHOB-PC). The experiment was performed in triplicate for each sample and repeated three times. Appressorium formation rates were calculated by dividing the number of conidia with appressoria by the number of germinated conidia. ${ }^{* *} p<0.01$ (Student's $t$-test) compared with $\Delta c b p 1$. Error bars indicate standard error.

in these mutants and the wild type strain. In the $C B P 1$-complemented strain, appressorium formation levels were significantly increased compared with the $\Delta c b p 1$ mutant. By contrast, in the inactive mutant Cbp1-D161A, appressorium formation appeared the same as in the $\Delta c b p 1$ mutant (Fig. 2(b)). We similarly generated substitution mutants of the second aspartate residue in the (a) domain and another motif (RPPY, Fig. 1(b)), and observed similar phenotypes (Fig. S2). These results suggested that the CDA activity of Cbp1 plays an important role in appressorium formation on a solid surface made of hydrophobic polyvinyl chloride (PHOB-PC).

Other CDA genes could not fully complement the function of Cbp1. M. oryzae has seven homologous CDA genes including $C B P 1^{32}$. We reasoned that the other gene(s) might compensate for a loss of Cbp1 function. We therefore compared their sequences using CLUSTAL W, but they did not share strong homology with each other. We focused on two Cbp1 like proteins, MGG_09159 (chitin-deacetylase, which we named Cbp1 like protein 1, Cbl1) and MGG_14966 (a hypothetical protein, which we named Cbl2), which resembled Cbp1 at the domain level (Fig. 3(a)). Cbl1 and Cbl2 shared two chitin-binding domains similar to those of Cbp1, but their positions were different from those in Cbp1.

We compared the expression patterns of the seven CDA homologues between the wild type and $\Delta c b p 1$ at 3 and 6 hpi (Fig. 3(b)). Differentiation of appressoria was initiated in the wild type at 3 hpi and in $\Delta c b p 1$ at 6 hpi. In the wild type, $C B L 6$ was upregulated at 6 hpi compared with 3 hpi but the expression of the other genes did not change between 3 and 6 hpi. In $\triangle c b p 1, C B L 2$ was downregulated and $C B L 4$ was upregulated regardless of time point. Interestingly, in $\triangle c b p 1, C B L 6$ was downregulated at $3 \mathrm{hpi}$ and upregulated at $6 \mathrm{hpi}$. These results indicated that $C B L 6$ might act in a later phase of appressorium differentiation and that $C B L 4$ was upregulated by $C B P 1$ deletion, but could not completely compensate for the loss of Cbp1 function.

At $6 \mathrm{hpi}$ after adding HDD, appressorium formation in $\Delta c b p 1$ occurred at the same rate as in the wild type. Thus, we compared the expression patterns of the seven CDA homologues at 3 hpi with or without HDD (Fig. 3(c)). In the wild type, CBL5 was downregulated and CBL6 was upregulated by adding HDD. In $\triangle c b p 1$, all genes except $C B L 2$ were upregulated by adding HDD, and $C B L 2$ also appeared to be upregulated although the change was not significant. These results suggested that the upregulation of all $C B L$ s may compensate for the deletion of $C B P 1$. 
(a)

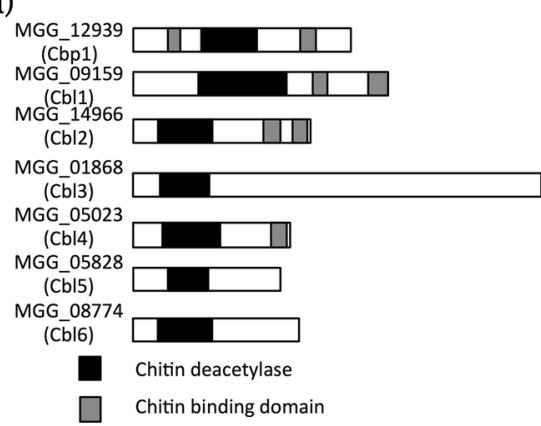

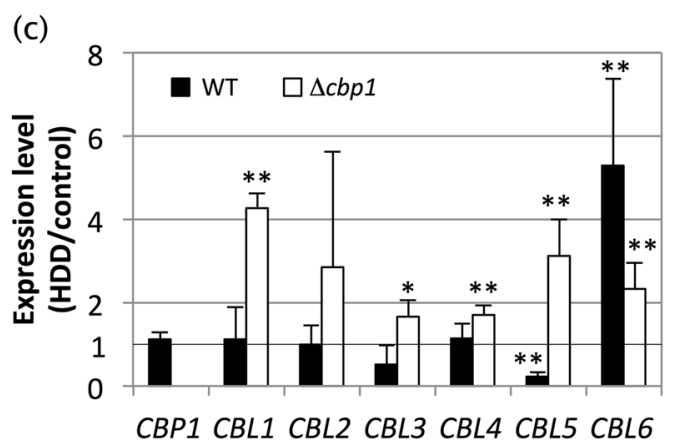

(b)

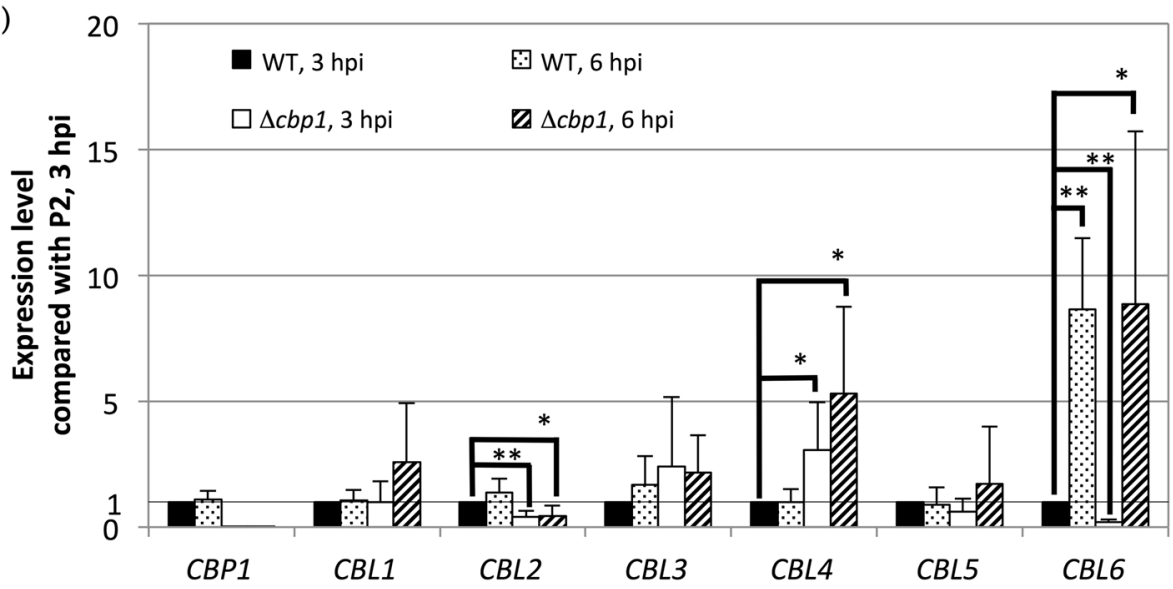

Figure 3. Analyses of CDA homologous proteins. (a) The domain features of seven CDA-homologous proteins. Black boxes indicate homology to CDA, dark grey boxes indicate chitin binding domains. (b) and (c) show transcriptomic analyses of the seven CDA-homologous genes. The expression levels of $C B P 1$ and the other CDA-homologous genes were detected by real-time PCR. Each cDNA was reverse transcribed from RNA extracted from germinated conidia $(3 \mathrm{hpi}$ ) or appressoria ( $6 \mathrm{hpi})$. The expression levels were normalized by the expression level of HPRT in each strain. Real-time PCR experiments were repeated at least four times from each RNA preparation to confirm reproducibility. (b) Time-lapse analysis at 3 and 6 hpi. The expression values for each gene were normalized to the expression in the WT at $3 \mathrm{hpi}$. Expression levels $<1$ indicate lower expression than in the WT at 3 hpi and levels $>1$ indicate higher expression than in the WT at 3 hpi. $* p<0.05$, $* * p<0.01$ (Student's $t$-test) compared with the WT at 3 hpi. Error bars indicate standard deviation. (c) Effect of 1,16-hexadecanediol (HDD). Expression levels are shown as ratios compared with the expression level in each strain without HDD as a control. Numerical values $<1$ indicate downregulation and values $>1$ indicate upregulation by HDD addition. ${ }^{*} p<0.05,{ }^{* *} p<0.01$ (Student's $t$-test) compared with each control. Error bars indicate standard deviation.

We next investigated the role of Cbl1 in appressorium development in more detail. The cbl1 mutant strain did not show any visible defects in colony growth or morphology during appressorium differentiation. However, a $\Delta c b p 1 \Delta c b l 1$ double mutant strain showed a decrease in conidiation, conidial adhesion, and appressorium formation (Fig. S3). These results suggested that Cbl1 plays a minor role in appressorium formation at 6 hpi and acts synergistically with Cbp1.

Chitosan accumulation in the tips of germ tubes is important in inducing appressorium formation. Cbpl seemed to be involved in inducing appressorium formation, so we focused on chitosan in germ tubes. We stained chitosan at the cell surface using the chitosan-specific dye OGA ${ }^{488} 33$ during germ tube elongation. $\mathrm{OGA}^{488}$ is an oligogalacturonate coupled to Alexa Fluor 488 . $\mathrm{OGA}^{488}$ retains its natural affinity. It is impossible to observe the same conidium in consecutive sections because of the staining and washing procedures. Thus, in this study, we measured germ tube length as an indicator of the conidial development stage. In the wild type strain, fluorescence from $\mathrm{OGA}^{488}$ accumulated in the tips of germ tubes during germ tube elongation (Fig. 4(a) arrowhead). In contrast, very little fluorescence was detected in the tips of germ tubes of the $\Delta c b p 1$ mutant (Fig. 4(b), arrowhead). This result showed that chitosan was accumulated in the tips of germ tubes when M. oryzae was in the induction phase of appressorium formation.

\section{Discussion}

It was previously reported that $\mathrm{Cbp} 1$ is involved in signalling pathways associated with appressorium formation ${ }^{16}$, but how it behaves and the important role it plays were not clear. In this study, we investigated how Cbp1 works in forming an appressorium, focusing on the putative CDA activity of Cbp1. 


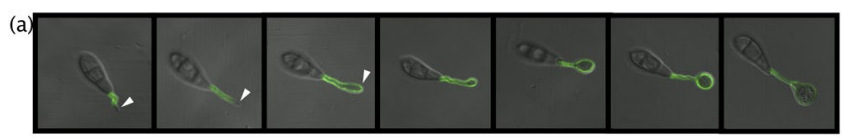

(b)

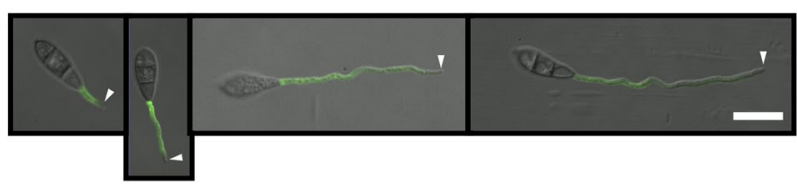

Figure 4. Time lapse of $\mathrm{OGA}^{488}$ fluorescence from conidia during germ tube elongation. $\mathrm{OGA}^{488}$ fluorescence was observed in the WT (a) and $\Delta c b p 1$ mutant (b). Conidia were placed on PHOB-PC for 1-6 hpi and stained with $\mathrm{OGA}^{488}$. We estimated germ tube length as an indicator of growth stage. Arrowheads show the tips of germ tubes. Bar $=20 \mu \mathrm{m}$.

We found that Cbp1 possesses CDA activity and that substitution mutations in the CDA active site cause a decrease in this activity. This is the first report of experimentally observed CDA activity in $M$. oryzae. In the $\triangle c b p 1$ mutant, $C B L 4$ was upregulated but did not compensate for the function of Cbp1. However, upregulation of all $C B L$ s was induced by the addition of HDD, which also induced appressorium differentiation at an early phase, as in the wild type. These results indicated that $\mathrm{Cbp} 1$ plays a main role in appressorium formation by converting chitin into chitosan during the early phase of appressorium differentiation. Furthermore, CBL6 was upregulated in both the wild type and $\Delta c b p 1$ by HDD addition, but the rate of increase was greater in the wild type than in the $\Delta c b p 1$ mutant. We reasoned that expression of $C B L 6$ during the early phase of appressorium differentiation might therefore be affected by Cbp1.

The relationship between CDA activity and pathogenicity has been investigated in some other pathogens. The pathogenic fungus Cladosporium fulvum, which causes leaf-mould disease in tomatoes, has an effector known as Ecp $^{34}$. Ecp6 contains LysM domains that have been shown to bind chitin. By binding chitin, LysM suppresses the elicitor activity of chitin and pathogen-associated molecular pattern triggered defence systems $\mathrm{s}^{35}$.

Geoghegan and Gurr ${ }^{18}$ proposed that Cbp1 does not function only in avoiding the host defence system because it seems to function as a CDA and is also involved in signalling. They showed that the presence of chitosan or the addition of exogenous chitosan at the cell surface of $M$. oryzae induced appressorium formation. However, they did not show the direct enzymatic detection of CDA activity of Cbp1.

In the case of phytopathogenic fungi, chitin may have become well masked during plant-microbe interactive co-evolution to avoid it acting as an elicitor and may no longer be a major defence target of plants. Our data suggested M. oryzae accumulates chitosan at the tips of germ tubes during induction of appressoria. We inferred that the amount of chitosan in the tips of germ tubes was sufficient and that the production of a sufficient amount of chitosan mainly depends on Cbp1 activity in the early phase of appressorium formation.

If the CDA activity of Cbp1 plays an important role in appressorium differentiation, other CDAs should be able to compensate for the loss of Cbp1. However, so far, we have not found any evidence that other CDAs can compensate for the $\Delta c b p 1$ mutation. We are now focusing on the localization of Cbp1 in detail. Cbp1 has a Ser/ Thr rich cluster and a putative GPI-anchoring signal as noted previously. These features strongly suggest that Cbp1 localizes to the surface of the cellular membrane or cell wall. Aside from Cbp1 and Cbl6, the other CDAs seem to be simple secreted proteins. We hypothesize that $M$. oryzae perceives signals from CDA activity with spatial-temporal exactness in the tips of germ tubes to activate the appressorium formation signalling pathway. Through these signals, $M$. oryzae may detect the conversion of chitin to chitosan under appropriate circumstances and conduct appressorium differentiation.

\section{Methods}

Fungal strains and culture conditions. M. oryzae wild type strain P2 was maintained as a stock culture in our laboratory and used as a wild type control throughout this study. Fungal strains were grown on oatmeal agar medium containing 5.0\% oatmeal (Quaker Oats Company, Chicago, IL, USA), 0.5\% sucrose (Nacalai Tesque, Kyoto, Japan) and $1.5 \%$ agar (Wako Pure Chemical Industries, Ltd., Osaka, Japan) at $28^{\circ} \mathrm{C}$. Conidiation was induced under BLB lamps (FL20S, Toshiba Co. Ltd, Tokyo, Japan) after removing the aerial hyphae with a sterilized brush. After 2-3 days of incubation under BLB lamps, the conidia were brushed off into distilled water. Mycelia for genomic DNA and RNA isolation and protoplast preparation were grown in YG liquid medium $(0.5 \%$ yeast extract (Nacalai Tesque) and $2.0 \%$ glucose (Nacalai Tesque)) with shaking at $28^{\circ} \mathrm{C}$.

CBP1 point mutation. A $\mathrm{KOD}^{+}$site directed mutagenesis kit (TOYOBO Co., Ltd., Osaka, Japan) was used to introduce point mutations. The $5^{\prime}$ termini of the primers were methylated using T4 Polynucleotide Kinase (Takara Bio Inc., Shiga, Japan) and pKS01 was amplified with the methylated primers using PCR $\left(95^{\circ} \mathrm{C}\right.$, $\left.2 \mathrm{~min} \rightarrow\left[95^{\circ} \mathrm{C}, 20 \mathrm{sec} ; 65^{\circ} \mathrm{C}, 30 \mathrm{sec} ; 68^{\circ} \mathrm{C}, 7 \mathrm{~min}\right] \times 5 \mathrm{cycles}\right)$. The CBP1_D161Af and CBP1_D161Ar primer pair was used to convert the first aspartate residue in the (a) domain (Fig. 1), CBP1_D162Af and CBP1_D161Ar for the second aspartate residue in the (a) domain (Fig. 1), and CBP1_R258Af and CBP1_R258Ar for the arginine in the (b) domain (Fig. 1). We transformed Escherichia coli DH5 $\alpha$ with the PCR product, which was digested by $\mathrm{DpnI}$ and ligated. Point mutations in $M$. oryzae were introduced using this vector. 
Heterologous expression in $E$. coli and CDA activity assay. Cbp1 and inactive Cbp1 were amplified by EcoRI_CBP1_F and SalI_CBP1_R and introduced into the pCold I vector (TakaRa) for transformation of $E$. coli Rosetta (F-, ompT, hsdSB (rB-mB-), gal, dcm (DE3) pRARE (CamR)) (Novagen, Madison, WI, USA). The primers used in this study are listed in Table S1. After incubation in LBA liquid medium $(0.1 \% \mathrm{NaCl}$ (Nacalai Tesque), $0.1 \%$ tryptone (Nacalai Tesque), $0.05 \%$ yeast extract, and $100 \mu \mathrm{g} / \mathrm{mL}$ ampicillin sodium salt (Nacalai Tesque)) at $37^{\circ} \mathrm{C}, 1.0 \mathrm{mM}$ IPTG was added and the culture was incubated for $24 \mathrm{~h}$ at $15^{\circ} \mathrm{C}$. The E. coli cells were then harvested by centrifugal separation and ultrasonication. Cbp1 was collected as an insoluble precipitate, because of inclusion body formation. The inclusion bodies were washed three times by ultrasonic fragmentation in washing buffer (20 mM Tris- $\mathrm{HCl}$ (pH 8.0; Nacalai Tesque), $2 \mathrm{mM} \mathrm{MgCl}_{2}$ (Nacalai Tesque), 2 units/mL benzonase endonuclease (Novagen) and $10 \mu \mathrm{g} / \mathrm{mL}$ lysozyme (Wako)). After washing, the precipitate was suspended with sterilized water to give a crude extract derived from $1 \times 10^{7}$ total bacterial count per $\mu \mathrm{L}$. CDA activity was measured by the MBTH method following the protocol previously reported ${ }^{36}$ and glycol chitin was synthesized from glycol chitosan (Wako) as previously reported ${ }^{37}$. Briefly, $100 \mu \mathrm{L}$ of $50 \mathrm{mM}$ sodium tetraborate buffer ( $\mathrm{pH}$ 8.5) (Nacalai Tesque), $100 \mu \mathrm{L}$ of $0.1 \%$ glycol chitin and $50 \mu \mathrm{L}$ of crude extract sample were mixed and incubated for $30 \mathrm{~min}$ at $37^{\circ} \mathrm{C}$. Then, $250 \mu \mathrm{L}$ of $2 \mathrm{~N} \mathrm{H}_{2} \mathrm{SO}_{4}$ was added to stop the enzyme reaction. After adding $250 \mu \mathrm{L}$ of $5 \%$ $\mathrm{NaNO}_{3}$ and incubating for $5 \mathrm{~min}$ at RT, $250 \mu \mathrm{L}$ of $12.5 \%$ ammonium sulfate was added and the mixture was incubated for $15 \mathrm{~min}$ at RT. Then, $250 \mu \mathrm{L}$ of $0.5 \% \mathrm{MBTH}$ (Tokyo Chemical Industry Co., Ltd., Tokyo, Japan) was added and the samples were boiled for 3 min and rapidly cooled. After that, $25 \mu \mathrm{L}$ of $5 \% \mathrm{FeCl}_{3}$ was added to develop a colour and the samples were incubated for $30 \mathrm{~min}$. CDA activity was measured by the absorbance at $650 \mathrm{~nm}$.

Extraction of genomic DNA and transformation of $\boldsymbol{M}$. oryzae. M. oryzae hyphae were incubated in $30 \mathrm{~mL}$ of YG liquid medium at $28^{\circ} \mathrm{C}, 150 \mathrm{rpm}$ for 2 days and then inoculated into $100 \mathrm{~mL}$ YG liquid medium and kept at $28^{\circ} \mathrm{C}, 150 \mathrm{rpm}$ for 1 day. Then, the fungal mycelium samples were harvested by centrifugation at $2000 \times g$ for $10 \mathrm{~min}$ and freeze-fractured. Extraction of genomic DNA from the fungal mycelia was performed following a previously described method ${ }^{38}$. Protoplasting and transformation of $M$. oryzae with the selectable markers BSD or $H P H$ were performed as described previously ${ }^{39}$.

Appressorium formation assay. Conidia were harvested from 5 to 7 -day-old cultures on oatmeal agar medium and adjusted to $3 \times 10^{4}$ conidia per $\mathrm{mL}$ with sterile distilled water. Drops of the conidia suspension $(20 \mu \mathrm{L})$ were placed on the surface of hydrophobic polyvinyl chloride (PHOB-PC) (Thermo Fisher Scientific, Inc., Waltham, MA, USA). The appressorium formation assays were performed at least three times with triplicate slides. The percentage of germinated conidia forming appressoria was observed by microscope at $6 \mathrm{~h}$ post inoculation (hpi). The appressorium formation rates were calculated as the number of germinated conidia with appressoria divided by the number of germinated conidia and the data were analysed statistically by Student's $t$-test. Chemicals were used as appressorium formation inducers; $10 \mu \mathrm{M}$ 1,16-hexadecanediol (HDD, (Tokyo Chemical Industry Co., Ltd.)) suspended in $0.1 \%$ ethanol.

Fluorescence analysis. To observe chitosan, appressorium formation was induced and the conidia with appressoria were stained with OGA ${ }^{488}$. Staining with OGA ${ }^{488}$ was performed as described previously ${ }^{33}$. After incubation, the samples were washed twice with $100 \mu \mathrm{L}$ of $25 \mathrm{mM}$ MES buffer, $\mathrm{pH}$ 5.7. Then, droplets of OGA ${ }^{488}$ were diluted 2000-fold with $25 \mathrm{mM}$ MES buffer, pH 5.7 and kept in the dark for $20 \mathrm{~min}$. The samples were washed twice with $100 \mu \mathrm{L}$ MES buffer before observation. We used an LSM 5 EXCITER (Carl Zeiss AG, Oberkochen, Germany), Ar laser (488 nm), HFT 488 beam splitter and 505-530 suppression filter for fluorescence observations.

Homology search. Protein sequences were obtained from National Center for Biotechnology Information (http://www.ncbi.nlm.nih.gov). We used BLAST ${ }^{40}$ for homology searches and CLUSTALW ${ }^{41}$ to compare amino acid sequences.

RNA extraction and real-time PCR. To obtain RNA from the germling stage, we induced appressorium formation on hydrophobic polycarbonate plates (SANPLATEC Co., Ltd, Osaka, Japan) instead of PHOB-PC. At $3 \mathrm{hpi}$, when some conidia were germinating, and at $6 \mathrm{hpi}$, when some conidia formed appressoria in the wild type, the germlings on polycarbonate plates were frozen with liquid nitrogen and continuously lyophilized in a vacuum freeze dryer FDU-2110 (TOKYO RIKAKIKAI Co., Ltd, Tokyo, Japan). Then, we collected the germinated conidia from the hydrophobic polycarbonate plates using a razor. RNA samples were extracted from the conidia using RNAzol (Cosmo Bio Co., Ltd., Tokyo, Japan) according to the manufacturer's protocol. To synthesize first-strand cDNA from the RNA, we used ReverTra Ace (TOYOBO Co., Ltd.) following the protocols of the kit. Real-time PCR was performed using SYBR green on a 7300 Real-Time PCR instrument (Thermo Fisher Scientific, Inc.). The expression levels of $C B P 1$ and the $C B L$ s were normalized by the expression levels of internal standard, HPRT (hypoxanthine phosphoribosyl transferase: $M G G \_10052$ ). We used the primers CBP1rt14_S and CBP1rt14_AS for CBP1 detection, CBL1_up and CBL1_down for CBL1, CBL2_up and CBL2_down for CBL2, CBL3_up3 and CBL3_down3 for CBL3, CBL4_up and CBL4_down for CBL4, CBL5_up and CBL5_down for CBL5, CBL6_up2 and CBL6_down2 for CBL6, and HPRT up and HPRT down for HPRT.

\section{References}

1. Food and Agriculture Organization of the United Nations. Record cereal production to lift global inventories to an all-time high http:// www.fao.org/worldfoodsituation/csdb/en/ (2017).

2. Kumar, M. K. P. et al. Fungicides - Showcases of Integrated Plant Disease Management from Around the World. Impact of Fungicides on Rice Production in India. (ed. Nita M.) 77-98 (Agricultural and Biological Sciences: In tech, 2013).

3. Hamer, J. E., Howard, R. J., Chumley, F. G. \& Valent, B. A mechanism for surface attachment in spores of a plant pathogenic fungus. Science 239(4837), 288-290 (1988). 
4. Valent, B. \& Chumley, F. G. Molecular genetic analysis of the rice blast fungus, Magnaporthe grisea. Annual Rev Phytopathol 29(1), 443-467 (1991)

5. Bourett, T. M. \& Howard, R. J. In vitro development of penetration structures in the rice blast fungus Magnaporthe grisea. Can. J. Bot 68(2), 329-342 (1990).

6. Xu, J. R. \& Hamer, J. E. MAP kinase and cAMP signaling regulate infection structure formation and pathogenic growth in the rice blast fungus Magnaporthe grisea. Genes Dev 10(21), 2696-2706 (1996).

7. Zhao, X., Mehrabi, R. \& Xu, J. R. Mitogen-activated protein kinase pathways and fungal pathogenesis. Eukaryot Cell 6(10), 1701-1714 (2007).

8. Howard, R. J., Ferrari, M. A., Roach, D. H. \& Money, N. P. Penetration of hard substrates by a fungus employing enormous turgor pressures. Proc Natl Acad Sci 88(24), 11281-11284 (1991).

9. Money, N. P. \& Howard, R. J. Confirmation of a link between fungal pigmentation, turgor pressure, and pathogenicity using a new method of turgor measurement. Fungal Genet Biol. 20(3), 217-227 (1996).

10. Dean, R. A. Signal pathways and appressorium morphogenesis. Annu Rev Phytopathol 35(1), 211-234 (1997).

11. Jelitto, T. C., Page, H. A. \& Read, N. D. Role of external signals in regulating the pre-penetration phase of infection by the rice blast fungus, Magnaporthe grisea. Planta 194(4), 471-477 (1994).

12. Lee, Y. H. \& Dean, R. A. Hydrophobicity of contact surface induces appressorium formation in Magnaporthe grisea. FEMS Microbiol Lett 115(1), 71-75 (1994).

13. Liu, X. H., Lu, J. P. \& Lin, F. C. Autophagy during conidiation, conidial germination and turgor generation in Magnaporthe grisea. Autophagy 3(5), 472-473 (2007).

14. Xiao, J. Z., Watanabe, T., Kamakura, T., Ohshima, A. \& Yamaguchi, I. Studies on cellular differentiation of Magnaporthe grisea. Physicochemical aspects of substratum surfaces in relation to appressorium formation. Physiol. Mol. Plant Pathol 44(3), 227-236 (1994).

15. Gilbert, R. D., Johnson, A. M. \& Dean, R. A. Chemical signals responsible for appressorium formation in the rice blast fungus Magnaporthe grisea. Physiol. Mol. Plant Pathol 48(5), 335-346 (1996).

16. Kamakura, T., Yamaguchi, S., Saitoh, K. I., Teraoka, T. \& Yamaguchi, I. A novel gene, CBP1, encoding a putative extracellular chitinbinding protein, may play an important role in the hydrophobic surface sensing of Magnaporthe grisea during appressorium differentiation. MPMI 15(5), 437-444 (2002).

17. Lee, Y. H. \& Dean, R. A. cAMP regulates infection structure formation in the plant pathogenic fungus Magnaporthe grisea. Plant Cell 5(6), 693-700 (1993).

18. Geoghegan, I. A. \& Gurr, S. J. Chitosan Mediates Germling Adhesion in Magnaporthe oryzae and Is Required for Surface Sensing and Germling Morphogenesis. PLoS Pathog 12(6), e1005703 (2016).

19. Talbot, N. J., Ebbole, D. J. \& Hamer, J. E. Identification and characterization of MPG1, a gene involved in pathogenicity from the rice blast fungus Magnaporthe grisea. Plant Cell 5(11), 1575-1590 (1993).

20. Romano, J. et al. Disruption of the Aspergillus fumigatus ECM33 homologue results in rapid conidial germination, antifungal resistance and hypervirulence. Microbiology 152(7), 1919-1928 (2006).

21. Plaine, A. et al. Functional analysis of Candida albicans GPI-anchored proteins: roles in cell wall integrity and caspofungin sensitivity. Fungal Genet Biol 45(10), 1404-1414 (2008).

22. Bartnicki-Garcia, S. Cell wall chemistry, morphogenesis, and taxonomy of fungi. Annu Rev Microbiol 22(1), 87-108 (1968).

23. Minke, R. \& Blackwell, J. The structure of alpha chitin. J Mol Biol 120, 167-181 (1978).

24. Tsigos, I., Martinou, A., Kafetzopoulos, D. \& Bouriotis, V. Chitin deacetylases: new, versatile tools in biotechnology. Trends Biotechnol 18(7), 305-312 (2000).

25. Zhao, Y., Park, R. D. \& Muzzarelli, R. A. Chitin deacetylases: properties and applications. Mar Drugs 8(1), 24-46 (2010).

26. Blair, D. E. et al. Structure and mechanism of chitin deacetylase from the fungal pathogen Colletotrichum lindemuthianum. Biochemistry 45(31), 9416-9426 (2006).

27. Nahar, P., Ghormade, V. \& Deshpande, M. V. The extracellular constitutive production of chitin deacetylase in Metarhizium anisopliae: possible edge to entomopathogenic fungi in the biological control of insect pests. Journal of invertebrate pathology 85(2), $80-88(2004)$

28. Tsigos, I. \& Bouriotis, V. Purification and characterization of chitin deacetylase from Colletotrichum lindemuthianum. Journal of Biological Chemistry 270(44), 26286-26291 (1995).

29. Alfonso, C., Nuero, O. M., Santamaría, F. \& Reyes, F. Purification of a heat-stable chitin deacetylase from Aspergillus nidulans and its role in cell wall degradation. Curr Microbiol. 30(1), 49-54 (1995).

30. Yamada, M. et al. Isolation and characterization of a gene coding for chitin deacetylase specifically expressed during fruiting body development in the basidiomycete Flammulina velutipes and its expression in the yeast Pichia pastoris. FEMS Microbiol Lett 289(2), 130-137 (2008)

31. Martinou, A., Koutsioulis, D. \& Bouriotis, V. Expression, purification, and characterization of a cobalt-activated chitin deacetylase (Cda2p) from Saccharomyces cerevisiae. Protein expression and purification 24(1), 111-116 (2002).

32. Mochizuki, S., Saitoh, K. I., Minami, E. \& Nishizawa, Y. Localization of probe-accessible chitin and characterization of genes encoding chitin-binding domains during rice-Magnaporthe oryzae interactions. J Gen Plant Pathol 77(3), 163-173 (2011).

33. Mravec, J. et al. Tracking developmentally regulated post-synthetic processing of homogalacturonan and chitin using reciprocal oligosaccharide probes. Development 141(24), 4841-4850 (2014).

34. Bolton, M. D. et al. The novel Cladosporium fulvum lysin motif effector Ecp6 is a virulence factor with orthologues in other fungal species. Mol Microbiol 69(1), 119-136 (2008).

35. de Jonge, R. et al. Conserved fungal LysM effector Ecp6 prevents chitin-triggered immunity in plants. Science 329(5994), 953-955 (2010).

36. Trudel, J. \& Asselin, A. Detection of chitin deacetylase activity after polyacrylamide gel electrophoresis. Analytical biochemistry 189(2), 249-253 (1990).

37. Trudel, J. \& Asselin, A. Detection of chitinase activity after polyacrylamide gel electrophoresis. Analytical biochemistry 178(2), 362-366 (1989)

38. Chi, M. H., Park, S. Y. \& Lee, Y. H. A quick and safe method for fungal DNA extraction. Plant Pathol. J 25(1), 108-111 (2009).

39. Kimura, M. et al. A novel transformation system for Pyricularia oryzae: adhesion of regenerating fungal protoplasts to collagencoated dishes. Biosci. Biotechnol. biochem 59(6), 1177-1180 (1995).

40. Altschul, S. F., Gish, W., Miller, W., Myers, E. W. \& Lipman, D. J. Basic local alignment search tool. J Mol Biol 215(3), 403-410 (1990).

41. Thompson, J. D., Higgins, D. G. \& Gibson, T. J. CLUSTAL W: improving the sensitivity of progressive multiple sequence alignment through sequence weighting, position-specific gap penalties and weight matrix choice. Nucleic Acids Res 22(22), 4673-4680 (1994).

\section{Acknowledgements}

We thank William G.T. Willats and Jozef Mravec for providing OGA ${ }^{488}$ and wish to acknowledge Professor Nicholas J Talbot, University of Exeter, for his help in discussion and advice about this study. We thank Robbie Lewis, MSc, from Edanz Group (www.edanzediting.com/ac) for editing a draft of this manuscript. 


\section{Author Contributions}

M.K. wrote manuscript. M.K., K.O., S.Y., Y.O., S.M., Y.N., A.N., N.T., M.N., H.T., K.S., T.T. and M.N. acquired the data. T.K. designed and conducted this study.

\section{Additional Information}

Supplementary information accompanies this paper at doi:10.1038/s41598-017-10322-0

Competing Interests: The authors declare that they have no competing interests.

Publisher's note: Springer Nature remains neutral with regard to jurisdictional claims in published maps and institutional affiliations.

(c) Open Access This article is licensed under a Creative Commons Attribution 4.0 International License, which permits use, sharing, adaptation, distribution and reproduction in any medium or format, as long as you give appropriate credit to the original author(s) and the source, provide a link to the Creative Commons license, and indicate if changes were made. The images or other third party material in this article are included in the article's Creative Commons license, unless indicated otherwise in a credit line to the material. If material is not included in the article's Creative Commons license and your intended use is not permitted by statutory regulation or exceeds the permitted use, you will need to obtain permission directly from the copyright holder. To view a copy of this license, visit http://creativecommons.org/licenses/by/4.0/.

(C) The Author(s) 2017 